\title{
Covalent Surface Functionalization of Boron Nitride Nanotubes Fabricated with Diazonium Salt
}

\author{
Zhujun Wang, ${ }^{1,2}$ Qian Li, ${ }^{1}$ Jinfeng Liu, ${ }^{1}$ Huayi Li $\left(\mathbb{D},{ }^{1}\right.$ and Shuirong Zheng ${ }^{2}$ \\ ${ }^{1}$ Beijing National Laboratory for Molecular Sciences, CAS Key Laboratory of Engineering Plastics, Institute of Chemistry, \\ Chinese Academy of Sciences, Beijing 100190, China \\ ${ }^{2}$ Key Laboratory of Space Applied Physics and Chemistry, Ministry of Education, Northwestern Polytechnical University, \\ Xian, Shaanxi 710129, China
}

Correspondence should be addressed to Huayi Li; lihuayi@iccas.ac.cn

Received 12 December 2017; Revised 3 March 2018; Accepted 12 March 2018; Published 18 April 2018

Academic Editor: Yasuhiko Hayashi

Copyright (C) 2018 Zhujun Wang et al. This is an open access article distributed under the Creative Commons Attribution License, which permits unrestricted use, distribution, and reproduction in any medium, provided the original work is properly cited.

\begin{abstract}
The chemical inertness and poor wetting properties of boron nitride nanotubes (BNNTs) hindered their applications. In this work, BNNTs have been functionalized with aniline groups by reacting with diazonium salt and the graft content of aniline component was calculated as high as $71.4 \mathrm{wt} . \%$. The chemical structure, composition, and morphology of functionalized BNNTs were carefully characterized to illustrate the modification. The anilinocarbocation generated by decomposition of diazonium salt reacted not only with $\mathrm{NH}_{2}$ sites, but also with B-OH sites on the surface of BNNTs. Meanwhile, the reaction applied a hot strong acid environment, which would help to open parts of B-N bonds to produce more reactive sites and enrich the functional groups grafted on the surface of BNNTs. Consequently, the functionalized BNNTs exhibited significantly improved dispersion stability in chloroform compared with pristine BNNTs. Amino surface functionalization of BNNTs offered more possibilities for surface chemical design of boron nitride and its practical application.
\end{abstract}

\section{Introduction}

As a structural analogue to carbon nanotubes (CNTs) [1], boron nitride nanotubes (BNNTs) containing boron and nitrogen atoms distributed equally in hexagonal rings have attracted much attention due to their excellent mechanical properties, high thermal conductivity, superb thermal and antioxidation stabilities, excellent biocompatibility, and effective light emission properties [2-5]. These make BNNTs great potential to be applied in many aspects, such as semiconductor devices and thermal conductive composite that work under high temperature, as well as photoelectric and neutron radiation shield materials [6-9].

Despite the advantages mentioned above, there are still some problems of BNNTs that hinder their further application. For one thing, BNNTs are extremely difficult to dissolve in organic solvents and water and are almost insoluble in all acids, bases, and solvents, so their solubility and dispersibility have greatly limited their application. For another, experimental modifications of BNNTs are much fewer than theoretical investigations due to their chemical inertness and poor wetting properties [10]. However, BNNTs can disperse well in certain solvent and polymer matrix after surface modification, which would expand their potential applications [11]. To this end, the development of new methods to realize the modification of BNNTs with functional groups attracted more and more attention.

To date, a variety of chemical methods by means of weak interactions and covalent bonding have been suggested to develop functionalized and dispersible BNNTs. Ciofani et al. implied the chemical reaction of the residual amino on the surface of the BNNTs and octadecyl chloride was used to make it graft long molecule chains, and the BNNTs after grafting could be dispersed in many solvents [12]. Maguer et al. used dimethyl sulfoxide to weaken the B-N bonds through cycloaddition, which make the part of B-N bonds on the surface of BNNTs hydrolysis and stripping the stripping $\mathrm{BN}$ molecular layer will be embedded in the BNNTs to form $\mathrm{Y}$ loop structure BNNTs [13]. The localized ionization structure enabled BNNTs to interact with substrates through stacking interactions and electrostatic interactions $[10,14,15]$. Actually, the covalent bonding chemistry of BNNTs, which could 
react at either $\mathrm{N}$ sites or $\mathrm{B}$ sites to achieve surface modification of BNNTs, has not been studied until recent years [16, 17]. The $\mathrm{N}$ site reaction mainly relied on the reactivity of surface amino group; however, there were usually few amino groups on the surface of BNNTs $[18,19]$. Ikuno et al. developed a method to increase concentration of active $\mathrm{N}$ sites through $\mathrm{NH}_{3}$ plasma treatment, but the surface damage cannot be avoided [20]. On the other hand, since most of the organic reactions are based on the $\mathrm{C}$ and $\mathrm{N}$ species, studies on $\mathrm{B}$ site reaction were not as sufficient [21]. Various chemical functionalization could be achieved by esterification between hydroxyl and carboxyl groups when B-site-activated BNNTs were used as the starting materials. Zhi et al. found that $\mathrm{B}$ sites could be activated by oxidizing BNNTs in $\mathrm{H}_{2} \mathrm{O}_{2}$ at high temperature and high pressure conditions [22]. Hydroxyl groups were attached on B sites of BNNTs and the hydroxylated BNNTs could be well dispersed in water opposed to pristine BNNTs. However, several problems have yet to be addressed. The major issue is represented by the lack of an effective method to graft a high density of functional groups on BNNTs and thus make BNNTs chemically active, which could allow for further efficient surface modification [23]. In addition, the understanding of the reaction mechanisms and the development of new functionalization methods for BNNTs are also challenging tasks.

In this work, we have proposed an innovative and simple approach to achieve chemically functionalized boron nitride nanotubes, which were abundantly decorated by aniline groups through the hydrolysis of the diazonium salt. The mechanism of the reaction was also studied, which suggested that the high active anilinocarbocations generated by the hydrolysis of diazonium salt reacted not only with $\mathrm{N}$ active point, but also with B active point on the surface of BNNTs. Therefore, the surface functionalized BNNTs could be greatly dispersed in the solvent of chloromethane, which greatly improves the compatibility with organic solvents.

\section{Materials and Methods}

2.1. Materials. Multiwalled boron nitride nanotubes (BNNTs) were purchased from Boron Nitride Research Center of Hebei University of Technology. The reagents used for the synthesis of diazonium chloride, including p-phenylenediamine, sodium nitrite $\left(\mathrm{NaNO}_{2}\right)$, concentrated hydrochloric acid $(\mathrm{HCl})$, and iron powder were purchased from J\&K Scientific Ltd. Other chemicals and reagents were used directly without further purification. Deionized water was generated by a Milli-Q integral ultrapure water purification system.

2.2. Sample Preparation. The synthesis of $\mathrm{NH}_{2} \mathrm{Ph}-\mathrm{BNNTs}$ was shown in Figure 1. The first step was the synthesis of diazonium chloride by using a modified diazotization reaction, followed by the hydrolysis of the diazonium salt and the introduction of aniline groups on the surface of BNNTs. Detailed procedures were given as follows. Firstly, $1.6 \mathrm{~g}$ of $\mathrm{p}$ phenylenediamine was dissolved in $20 \mathrm{~mL}$ of deionized water and then $11 \mathrm{~mL}$ of $0.5 \mathrm{~mol} \cdot \mathrm{mL}^{-1} \mathrm{HCl}$ was added dropwise under stirring within $30 \mathrm{~min}$ to form amaranth suspension.
Then the suspension was cooled in ice bath $\left(<5^{\circ} \mathrm{C}\right)$, and $1.02 \mathrm{~g}$ of $\mathrm{NaNO}_{2}$ dissolved in aqueous solution was slowly added under stirring to generate the diazonium chloride, which was immediately added to BNNTs suspension under stirring within $30 \mathrm{~min}$ at room temperature. Then $1.2 \mathrm{~g}$ of ironic powder and $50 \mathrm{~mL}$ of $0.5 \mathrm{~mol} \mathrm{~mL}^{-1} \mathrm{HCl}$ were added to the mixture successively and stirred for $2 \mathrm{~h}$ to obtain the targeting product. The product was washed with deionized water and methanol successively, followed by the treatment of ultrasonic and centrifugation to remove the extra diazonium chloride. In order to ensure that the impurities were fully removed, in the process of further postprocessing, the product was extracted by refluxing ethanol for $4 \mathrm{~h}$ in a set of Soxhlet apparatus. The resultant product was dried at $60^{\circ} \mathrm{C}$ for $24 \mathrm{~h}$ in vacuum oven and named as $\mathrm{NH}_{2} \mathrm{Ph}$-BNNTs.

2.3. Characterization. Fourier transform infrared spectroscopy (FT-IR) analysis was carried out using a Perkin-Emler System 2000 infrared spectrum analyzer in the wave number range of $4000-650 \mathrm{~cm}^{-1}$ with $\mathrm{KBr}$ pellets. Thermogravimetric analysis (TGA) was carried out using Perkin-Elmer Pyris 1 and TGA 7 thermogravimetric analyzer in air atmosphere. The thermal behavior was characterized by temperature programming from $50^{\circ} \mathrm{C}$ to $700^{\circ} \mathrm{C}$ at a heating rate of $10^{\circ} \mathrm{C} \mathrm{min}{ }^{-1}$. The content of the grafted functional groups was calculated by thermogravimetric loss between $250^{\circ} \mathrm{C}$ and $600^{\circ} \mathrm{C}$. X-ray photoelectron spectroscopy (XPS) data were obtained with an ESCALab 220i-XL electron spectrometer from VG Scientific using $300 \mathrm{~W} \mathrm{Al} \mathrm{K \alpha}$ radiation. The base pressure is about $3 \times 10^{-9}$ mbar. The binding energies are referenced to the $\mathrm{Cls}$ line at $284.6 \mathrm{eV}$ from adventitious carbon. Elemental composition analysis was obtained using Flash EA 1112 elemental analyzer. Scanning electron microscope (SEM) image was taken on a JEOL S-4300F field emission scanning electron microscope at $10 \mathrm{kV}$. Transmission electron microscope (TEM) image was obtained using a JEOL (Japan) JEM-2200FS instrument with an accelerating voltage of $150 \mathrm{kV}$. Specimen of TEM was prepared by placing a droplet of water-diluted sample on a carbon-coated microscope grid and dried in air.

\section{Results and Discussion}

3.1. Dispersity Analysis. As a consequence, lots of organic aniline groups were irregularly grafted on the surface of BNNTs, which could improve the dispersity of BNNTs in chloroform, as shown in Figure 2. The BNNTs and $\mathrm{NH}_{2} \mathrm{Ph}-\mathrm{BNNT}$ images captured by supersonic treatment followed by standing for $24 \mathrm{~h}$ in chloroform are shown in Figure 2. It is obvious that the obtained $\mathrm{NH}_{2} \mathrm{Ph}-\mathrm{BNNTs}$ were more stable than the pristine BNNTs in chloroform. The reason for the excellence dispersibility of $\mathrm{NH}_{2} \mathrm{Ph}-\mathrm{BNNT}$ is likely to be a result of the atomic degradation of the crystallinity [20]. These results suggested that aniline groups were attached on the surface of $\mathrm{NH}_{2} \mathrm{Ph}$-BNNTs and could increase the affinity between BNNTs and chloroform solvent.

3.2. FT-IR Analysis. Figure 3 shows the FT-IR spectra of BNNTs and $\mathrm{NH}_{2} \mathrm{Ph}-\mathrm{BNNTs}$. Main IR peaks corresponding to 


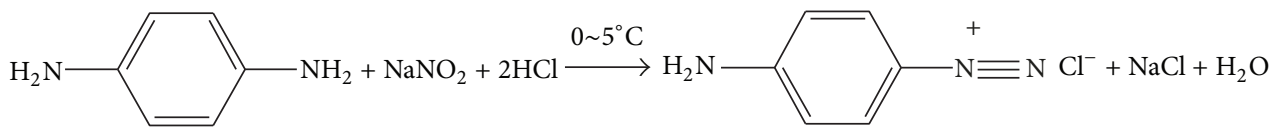

(a)
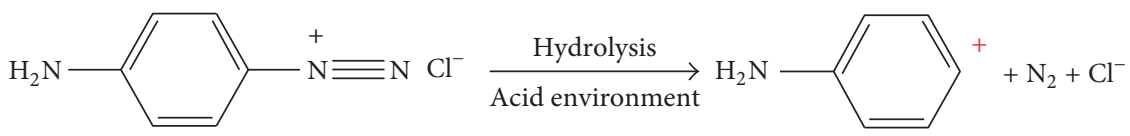

(b)
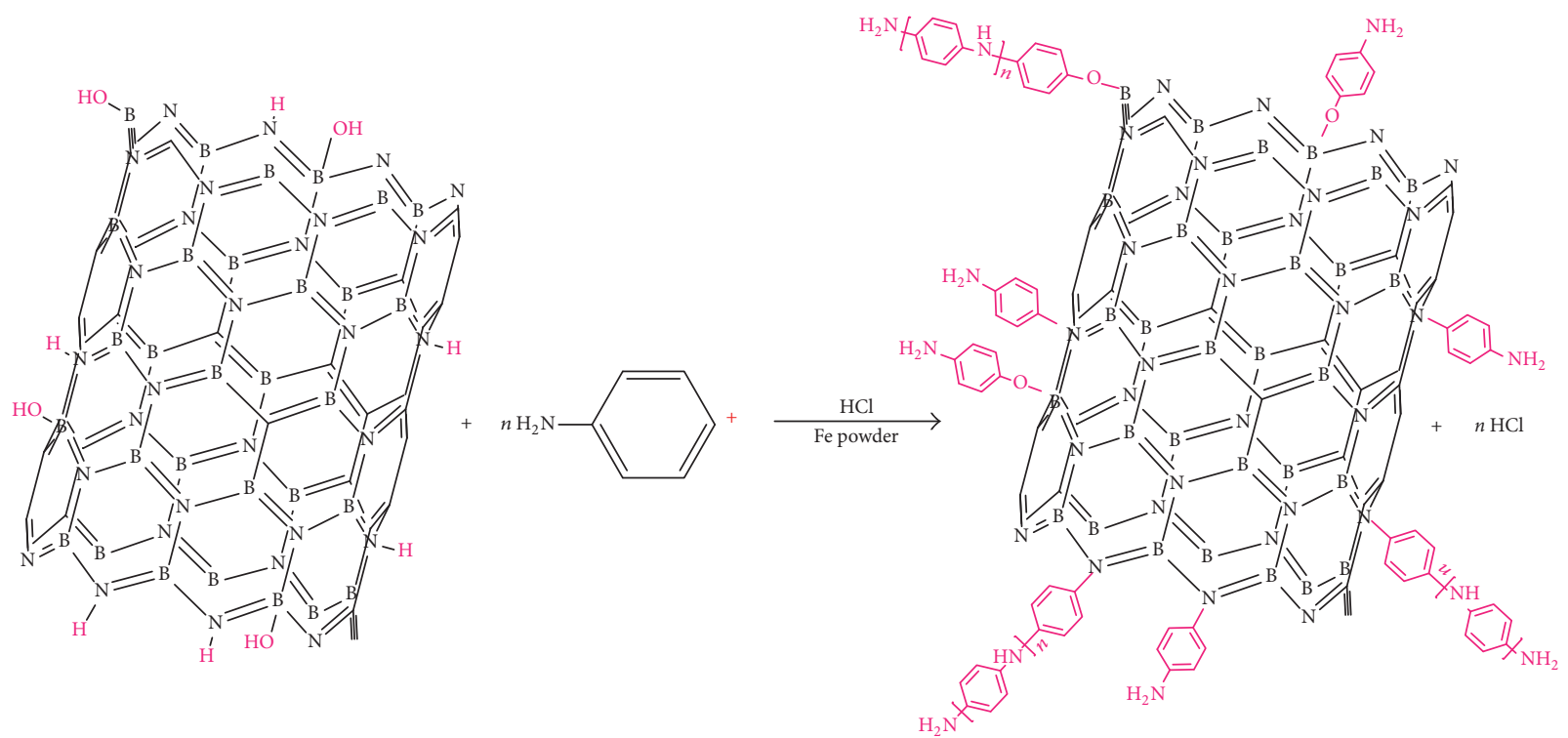

(c)

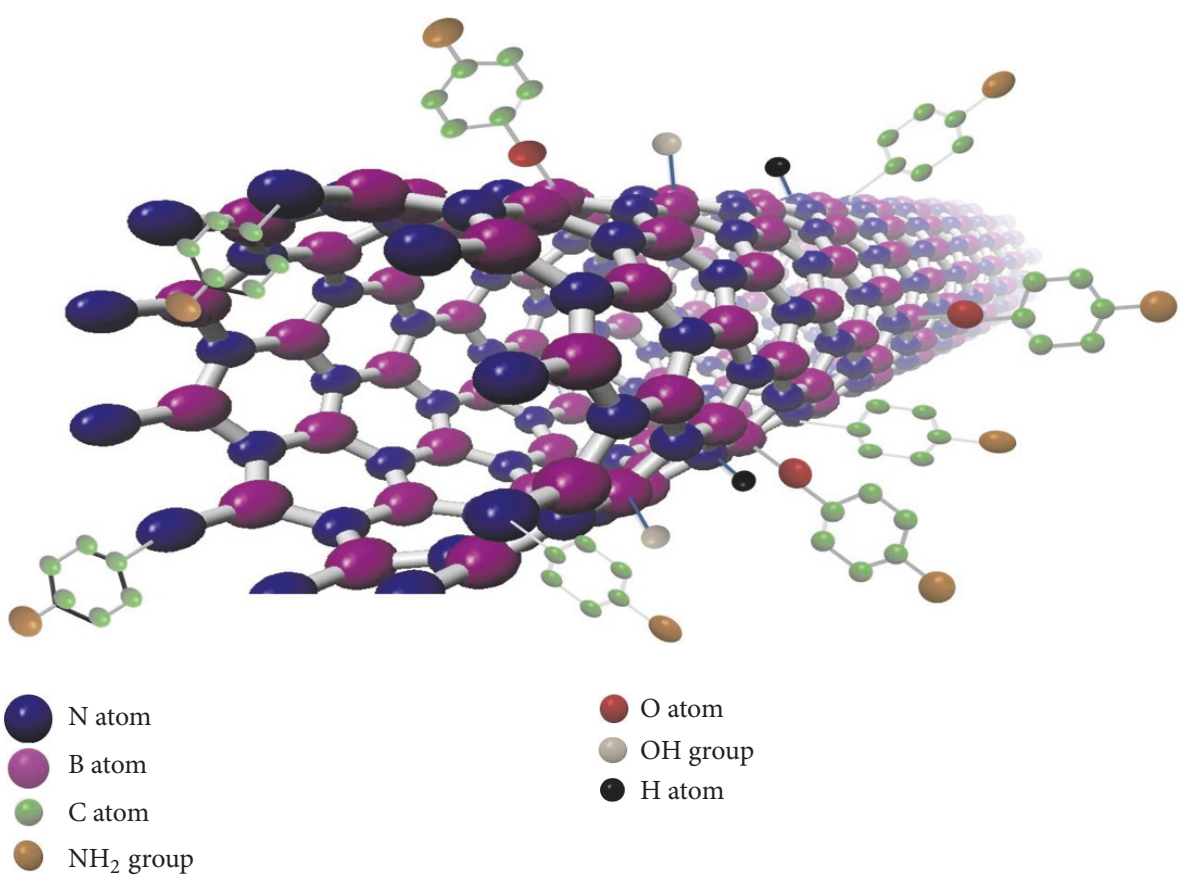

(d)

FIGURE 1: (a) The synthesis of diazonium salt chloride. (b) The hydrolysis of diazonium salt chloride. (c) The surface grafting between anilinocarbon cations and BNNTs. (d) The grafted schematic of single- $\mathrm{NH}_{2} \mathrm{Ph}-\mathrm{BNNTs}$. 


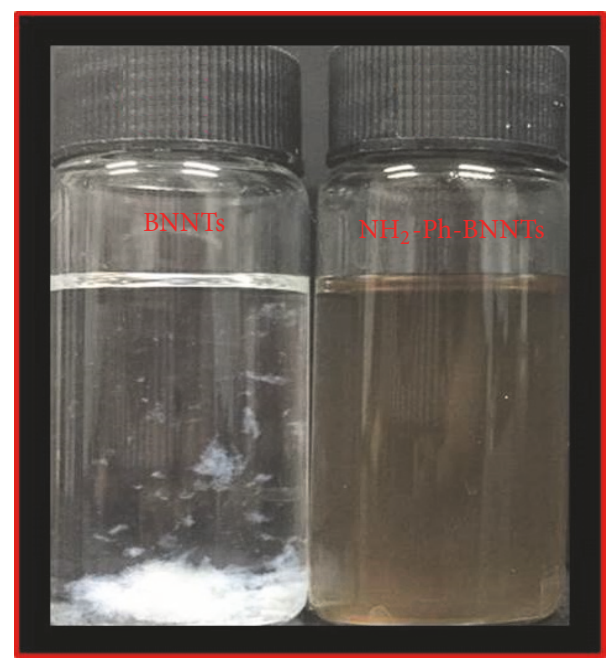

Figure 2: Photographs of vials containing the BNNTs and the $\mathrm{NH}_{2} \mathrm{Ph}-\mathrm{BNNTs}$ in chloroform after standing for $24 \mathrm{~h}$.

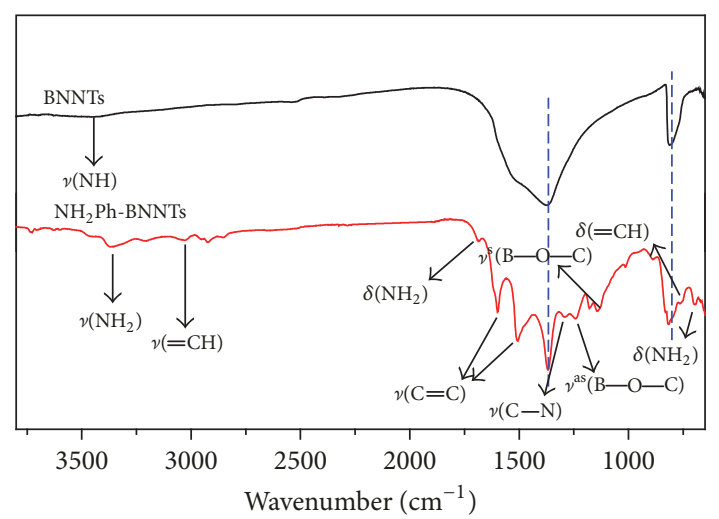

FIgURE 3: FT-IR spectra of BNNTs and $\mathrm{NH}_{2} \mathrm{Ph}-\mathrm{BNNTs}$.

literature values and their assignments were given in Table 1. Two main featured peaks were found for BNNTs sample, which were marked by two blue lines in the figure. The broad absorption band near $3424 \mathrm{~cm}^{-1}$ can be resulting from the $\mathrm{N}$ $\mathrm{H}$ bonds on the surface of BNNTs. These values were in good agreement with the reports concerning the FT-IR spectra of BNNTs [24, 25]. Compared with the spectra of BNNTs, some new peaks were observed in the spectra of $\mathrm{NH}_{2} \mathrm{Ph}$ BNNTs. These peaks indicated the existence of aniline groups in $\mathrm{NH}_{2} \mathrm{Ph}$-BNNTs. More importantly, the peaks at $1239 \mathrm{~cm}^{-1}$ and $1141 \mathrm{~cm}^{-1}$ were attributed to B-O-C bonds, which was the result of the reaction between anilinocarbocation and $\mathrm{B}-\mathrm{OH}$. This directly proved the success of grafting reaction. Moreover, slight blue shift of two featured peaks of $\mathrm{NH}_{2} \mathrm{Ph}$ BNNTs was also observed, which may result from the grafting of the electronegative aniline groups.

3.3. TGA Analysis. Furthermore, we utilized thermogravimetric analysis (TGA) to evaluate the grafting efficiency, as shown in Figure 4. The results of the thermogravimetric curves showed that there was almost no heat loss of BNNTs,
TABLE 1: The main characteristic peak values of BNNTs and $\mathrm{NH}_{2} \mathrm{Ph}$ BNNTs.

\begin{tabular}{lcc}
\hline $\begin{array}{l}\text { FT-IR peak values } \\
\left(\mathrm{cm}^{-1}\right)\end{array}$ & $\begin{array}{c}\text { Reference values } \\
\left(\mathrm{cm}^{-1}\right)\end{array}$ & Assignments \\
\hline 1324 & 1343 & $\beta_{\mathrm{B}-\mathrm{N}_{\mathrm{f}}}$ \\
783 & 789 & $\gamma_{\mathrm{B}-\mathrm{N}-\mathrm{B}}$ \\
3424 & $3500 \sim 3300$ & $\nu_{\mathrm{N}-\mathrm{H}}$ of amine group \\
697 & $750 \sim 600$ & $\gamma_{\mathrm{N}-\mathrm{H}}$ of amine group \\
3025 & $3100 \sim 3000$ & $\nu_{\mathrm{C}=\mathrm{H}}$ of benzene \\
1598 and 1507 & 1600 and 1500 & $\nu_{\mathrm{C}=\mathrm{C}}$ of benzene \\
1253 & $1340 \sim 1250$ & $\nu_{\mathrm{C}-\mathrm{N}}$ of aromatic \\
794 & $860 \sim 790$ & $\gamma_{\mathrm{C}=\mathrm{H}}$ of benzene \\
1239 and 1141 & $1300 \sim 900$ & $\nu_{\mathrm{sB}-\mathrm{O}-\mathrm{C}}$ and $\nu_{\text {asB-O-C }}$ \\
\hline
\end{tabular}

TABLE 2: The concentration of each element from XPS for BNNTs and $\mathrm{NH}_{2} \mathrm{Ph}-\mathrm{BNNTs}$.

\begin{tabular}{lcccc}
\hline \multirow{2}{*}{ Sample } & \multicolumn{4}{c}{ Chemical composition (at.\%) } \\
& B1s & N1s & C1s & O1s \\
\hline BNNTs & 52.55 & 44.65 & 1.84 & 0.96 \\
$\mathrm{NH}_{2} \mathrm{Ph}-\mathrm{BNNTs}$ & 2.08 & 3.88 & 81.1 & 12.93 \\
\hline
\end{tabular}

illustrating the thermal stable structure of BNNTs. On the contrary, obvious heat loss of $\mathrm{NH}_{2} \mathrm{Ph}-\mathrm{BNNTs}$ was observed. The weight loss of $\mathrm{NH}_{2} \mathrm{Ph}-\mathrm{BNNTs}$ mainly resulted from surface organic components, indicating the existence of organic ingredients in $\mathrm{NH}_{2} \mathrm{Ph}-\mathrm{BNNTs}$. Moreover, the graft content was calculated as $71.4 \mathrm{wt} . \%$. For one thing, the high grafting rate benefited from the broken of $\mathrm{B}-\mathrm{N}$ bonds on the surface of BNNTs in the acidic environment, producing more reactive sites. For another, we used largely excess diazonium chloride salt to decorate BNNTs. As we know, the excess diazonium salt could further react with the $\mathrm{Ph}-\mathrm{NH}_{2}$ group that has been grafted on the surface of BNNTs to form polyanilinelike oligomers. Therefore, this reaction would increase the grafting content. However, the high grafting content would significantly contribute to BNNTs' compatibility with organic substance.

3.4. XPS Analysis. XPS technique was applied to detect the chemical structures of BNNTs and $\mathrm{NH}_{2} \mathrm{Ph}-\mathrm{BNNTs}$. Figure 5 shows the wide-scan spectra of BNNTs and $\mathrm{NH}_{2} \mathrm{Ph}$-BNNTs. The element content data were calculated and summarized in Table 2. As shown in Figure 5, the peak intensity of Bls and $\mathrm{Nls}$ in $\mathrm{NH}_{2} \mathrm{Ph}$-BNNTs was obviously decreased compared to that of BNNTs. The decrease in the detectable boron and nitrogen elements was caused by the grafting layer on the surface of BNNTs. As mentioned above, polyaniline-like oligomers could form a coating layer, which would hinder the detection of the inner BNNTs. Actually, the electron microscope observation below would verify this point. In addition, due to the introduction of nitrogen carried by aniline groups, the N/B ratio of $\mathrm{NH}_{2} \mathrm{Ph}$-BNNTs was increased to 1.87 , which was much higher than that of BNNTs (0.85). 


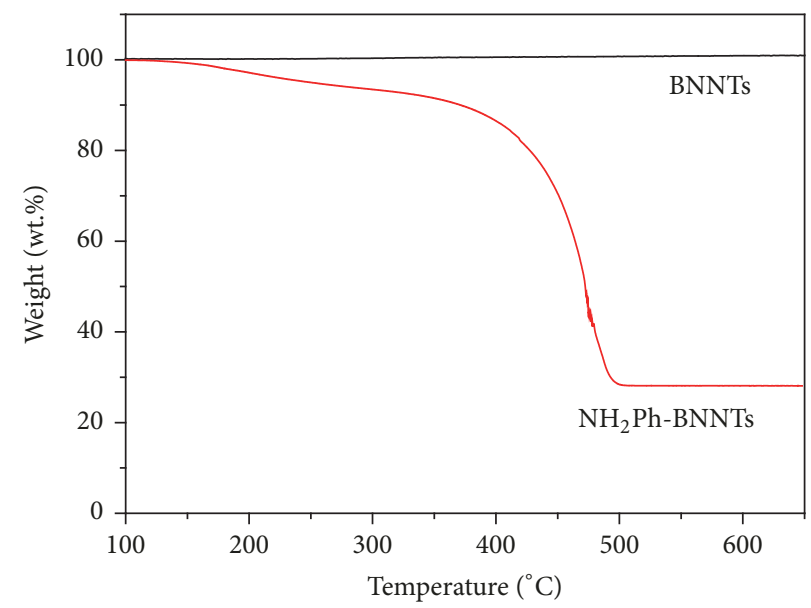

Figure 4: TGA curves of BNNTs and $\mathrm{NH}_{2} \mathrm{Ph}-\mathrm{BNNTs}$.

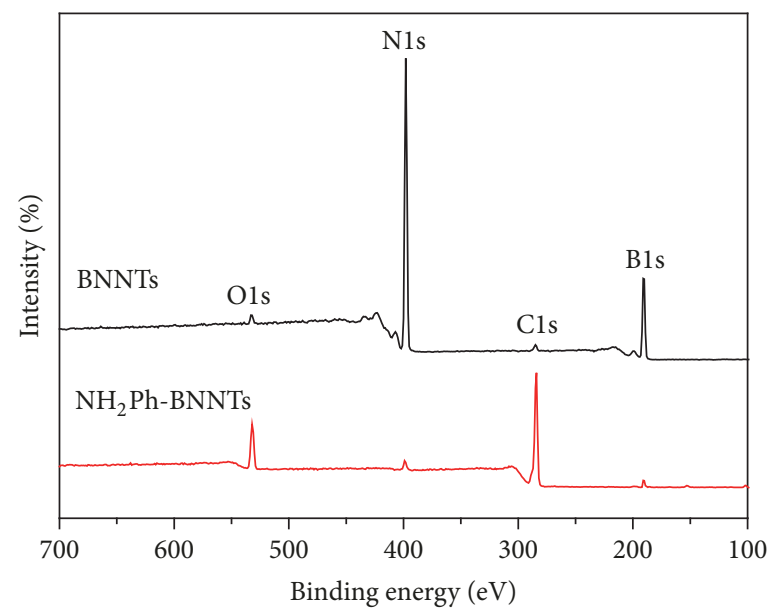

Figure 5: The wide-scan spectra of BNNTs and $\mathrm{NH}_{2} \mathrm{Ph}$-BNNTs.

The N1s photoelectron spectroscopy of BNNTs and $\mathrm{NH}_{2} \mathrm{Ph}-\mathrm{BNNTs}$ is shown in Figure 6. The N1s of BNNTs perfectly presented a single peak with binding energy at $397.9 \mathrm{eV}$, corresponding to B-N-B [26, 27]. Obviously, the N1s peak of $\mathrm{NH}_{2} \mathrm{Ph}$-BNNTs was not a single peak. It could be divided into four peaks by peak-fitting process located at $397.9 \mathrm{eV}, 399.4 \mathrm{eV}, 402.4 \mathrm{eV}$, and $400.8 \mathrm{eV}$, attributed to $\mathrm{B}-\mathrm{N}-\mathrm{B}$, $\mathrm{Ph}-\mathrm{NH}_{2}, \mathrm{C}-\mathrm{N}-\mathrm{B}$, and B-N-H, respectively [28]. These results indicated that the aniline groups existed in $\mathrm{NH}_{2} \mathrm{Ph}-\mathrm{BNNT}$. From the existence of $\mathrm{C}-\mathrm{N}-\mathrm{B}$ structure, we concluded that during the reaction anilinocarbocation also reacted with $\mathrm{N}-\mathrm{H}$ on the surface of BNNTs, in addition to reacting with $\mathrm{B}-\mathrm{OH}$ as FT-IR suggested.

The Bls photoelectron spectroscopy of BNNTs and $\mathrm{NH}_{2} \mathrm{Ph}-\mathrm{BNNTs}$ is shown in Figure 7. The peak at $190.3 \mathrm{eV}$ was found in both the BNNTs and $\mathrm{NH}_{2} \mathrm{Ph}$-BNNTs, which was assigned to B-N in BNNTs [24, 27]. There was another peak obtained from the peak-fitting; however, for this peak BNNTs and $\mathrm{NH}_{2}$ Ph-BNNTs showed slight different binding energy values. The peak at $191.4 \mathrm{eV}$ in BNNTs belonged to the - $\mathrm{B}-\mathrm{OH}$ while the peak at $191.9 \mathrm{eV}$ in $\mathrm{NH}_{2} \mathrm{Ph}-\mathrm{BNNTs}$ belonged to - $\mathrm{B}-\mathrm{O}$ - group. The replacement of $\mathrm{H}$ atom by electronegative aniline groups caused the rise of inner electron bonding energy of boron, and this was in accordance with the literatures results, simultaneously verifying the above FT-IR result $[6,19]$.

The O1s photoelectron spectroscopy of BNNTs and $\mathrm{NH}_{2} \mathrm{Ph}$-BNNTs is shown in Figure 8. The peak at $523.3 \mathrm{eV}$ corresponding to the - $\mathrm{B}-\mathrm{OH}$ group was found both in BNNTs and in $\mathrm{NH}_{2} \mathrm{Ph}$-BNNTs. Additionally, one peak assigned to B-O-C- group at about $533.3 \mathrm{eV}$ was also found in $\mathrm{NH}_{2} \mathrm{Ph}$ BNNTs, suggesting again that aniline was reacted with the $\mathrm{B}-\mathrm{OH}$ groups on the surface of BNNTs [19].

The Cls photoelectron spectroscopy of $\mathrm{NH}_{2} \mathrm{Ph}-\mathrm{BNNTs}$ is shown in Figure 9. The peak at $284.6 \mathrm{eV}$ caused by the contamination carbon in the raw materials was found both in the BNNTs and in $\mathrm{NH}_{2} \mathrm{Ph}-\mathrm{BNNTs}$. In addition, three peaks at $284.9 \mathrm{eV}, 286.5 \mathrm{eV}$, and $288.9 \mathrm{eV}$ were also observed in $\mathrm{NH}_{2} \mathrm{Ph}$-BNNTs and they were assigned to the -C-C-, -C-O-, and $-\mathrm{C}-\mathrm{N}$ - groups [28]. The existence of $-\mathrm{C}-\mathrm{O}-$ and $-\mathrm{C}-\mathrm{N}-$ indicated again that the aniline reacted with both $-\mathrm{B}-\mathrm{OH}$ and $-\mathrm{N}-\mathrm{H}$ groups on the surface of BNNTs. 

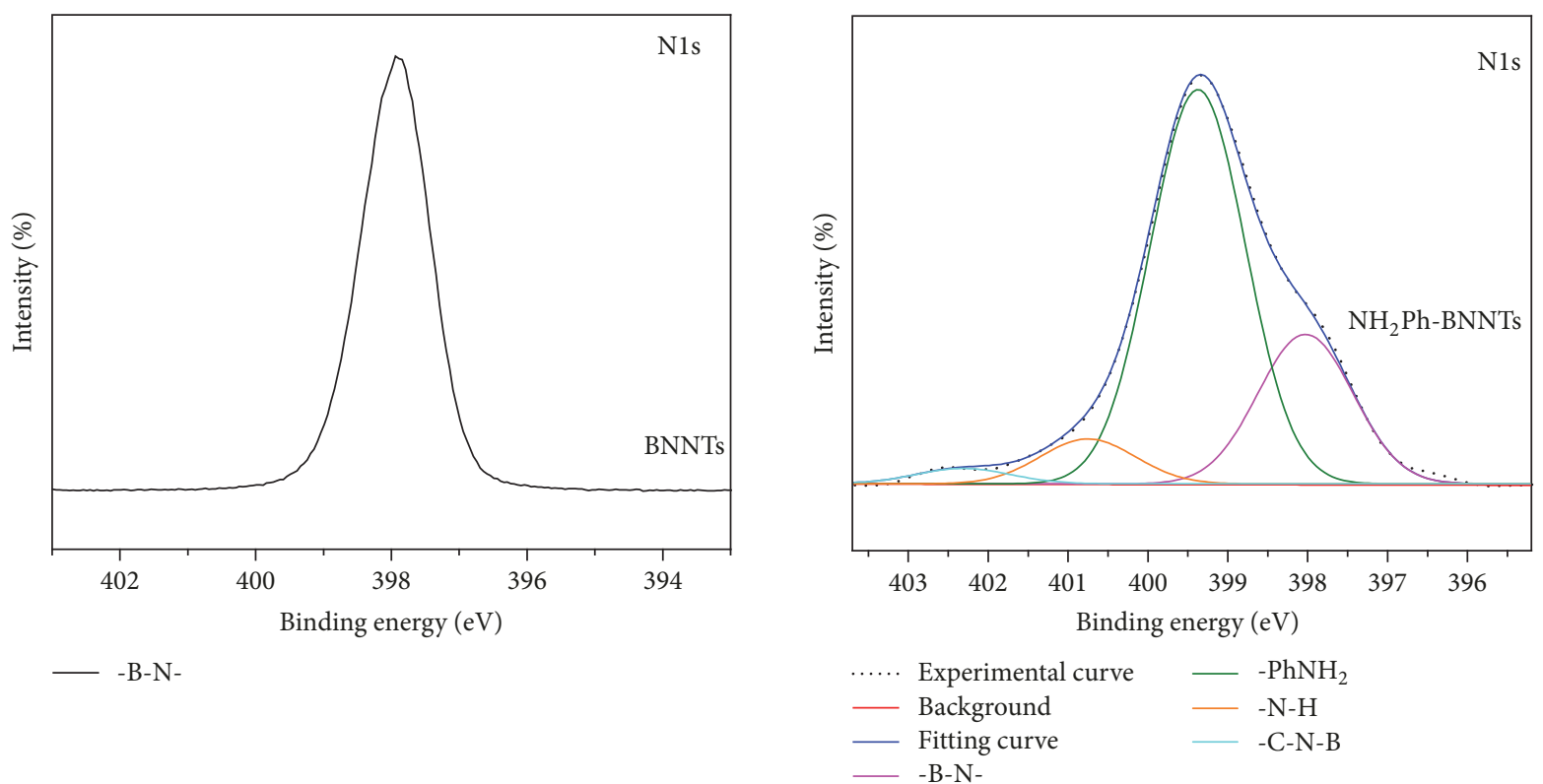

Figure 6: The N1s core level spectra of BNNTs and $\mathrm{NH}_{2}$ Ph-BNNTs.
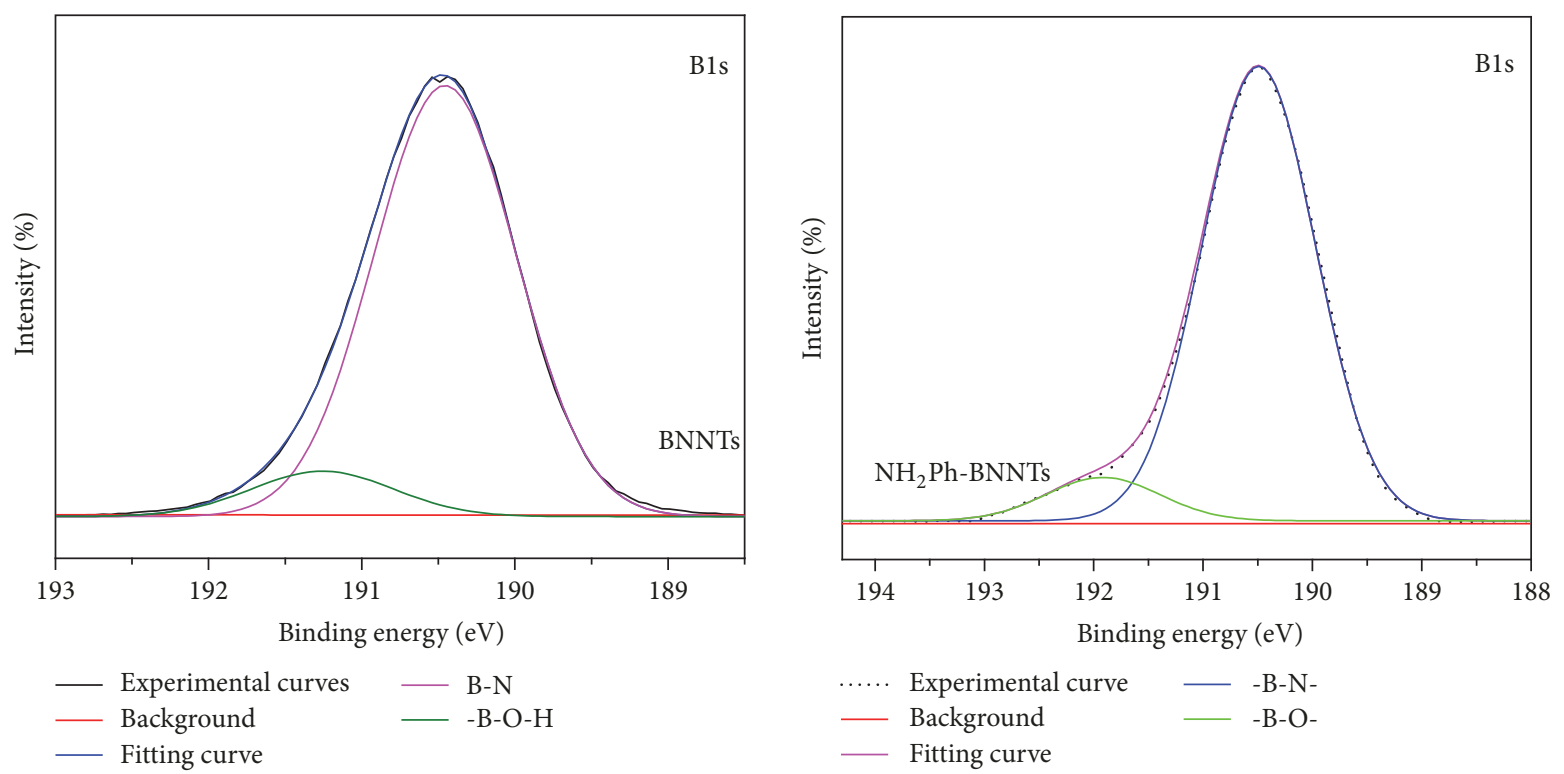

FIgURE 7: The B1s core level spectra of BNNTs and $\mathrm{NH}_{2}$ Ph-BNNTs.

3.5. Electron Microscope Analysis. The electron microscope presented the morphology of BNNTs and $\mathrm{NH}_{2} \mathrm{Ph}-\mathrm{BNNTs}$. SEM images of BNNTs were shown in Figures 10(a) and 10(b). The naked BNNTs were about dozens of micrometers in length, with diameter of $\sim 150 \mathrm{~nm}$ and their surfaces were smooth. After the grafting, amorphous organic coating layers were observed on the surface of nanotubes, introducing a rough surface of $\mathrm{NH}_{2} \mathrm{Ph}-\mathrm{BNNT}$. According to TEM images in Figures 10 (c) and 10(d), the coating layer was $15 \sim 25 \mathrm{~nm}$, but a single para-phenylenediamine molecule is about $1 \mathrm{~nm}$ in length. The difference between theory and actual thickness was too big, which might result from the largely excess diazonium chloride salts used to decorate BNNTs during the reaction. The excess diazonium salt could further react with the $\mathrm{Ph}-\mathrm{NH}_{2}$ group that has been grafted on the surface of BNNTs to form polyaniline-like oligomers. Therefore, the grafting reaction formed organic coating layer, wrapping the nanotube all up. Consequently, the surface structure and wetting properties of $\mathrm{NH}_{2} \mathrm{Ph}$-BNNTs became totally different from the naked BNNTs, just as discussed above. Moreover, Figure 10(b) captured one nanotube with part of BNNTs exposed, which presented the smooth surface of BNNTs, indicating there was no serious damage upon the integrity and tubular structure of BNNTs after the functionalization. 

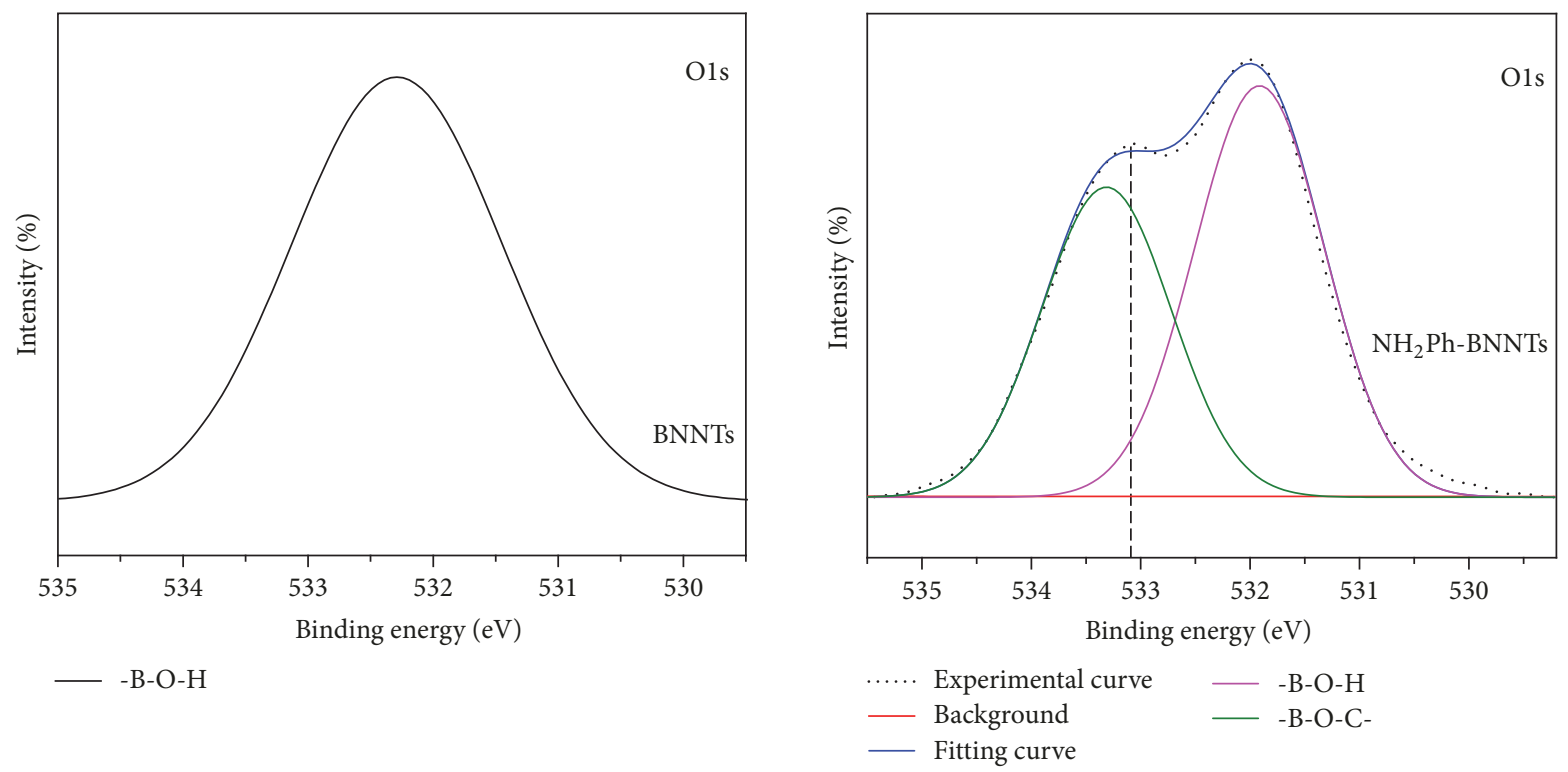

FIgUre 8: The O1s core level spectra of BNNTs and $\mathrm{NH}_{2}$ Ph-BNNTs.

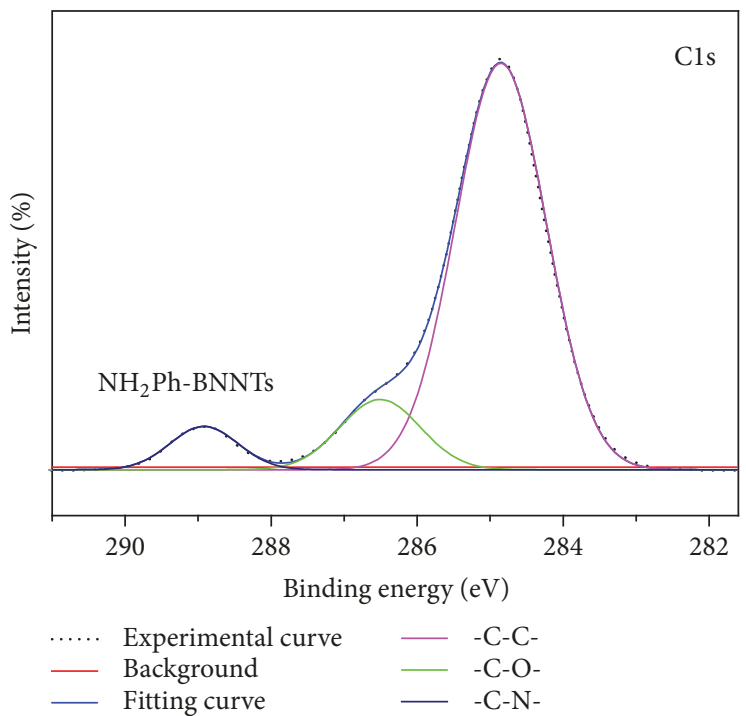

FIGURE 9: The Cls core level spectra of $\mathrm{NH}_{2} \mathrm{Ph}-\mathrm{BNNTs}$.

3.6. The Functionalization Mechanism. Finally, the functionalization mechanism was concluded as follows. At the first step diazonium chloride salt was synthesized. Then, in the BNNTs dispersion, diazonium salt chloride was decomposed to generate anilinocarbocations, which was active in acid environment. Meanwhile, under this strong acid environment, the surface B-N bonds of BNNTs were fractured to generate more $-\mathrm{B}-\mathrm{OH}$ and $-\mathrm{NH}$ groups $[25,29]$. Due to the high activity of anilinocarbocations, it could immediately capture the hydrogen atoms of $-\mathrm{NH}$ and $-\mathrm{B}-\mathrm{OH}$ groups on the surface of BNNTs. The grafting schematics were shown in Figures 1(c) and 1(d). The excess anilinocarbocations could react with the $\mathrm{Ph}-\mathrm{NH}_{2}$ that has been grafted on the surface of BNNTs to form polyaniline-like oligomers. There formed the coating layer upon the surface of BNNTs and the coating component was covalently bonded with BNNTs.

\section{Conclusion}

In this paper, we have proposed an innovative and simple approach to achieve chemically functionalized boron nitride nanotubes. High active anilinocarbocations generated by the hydrolysis of diazonium salt reacted both $\mathrm{N}-\mathrm{H}$ and $\mathrm{B}-\mathrm{OH}$ active sites on the surface of BNNTs. The hot acid aqueous environment helped to introduce more $\mathrm{N}-\mathrm{H}$ and $\mathrm{B}-\mathrm{OH}$ reactive sites. As a result, BNNTs were abundantly coated by organic polyaniline-like components and their dispersibility in organic solvent was greatly improved. It is expected that the 


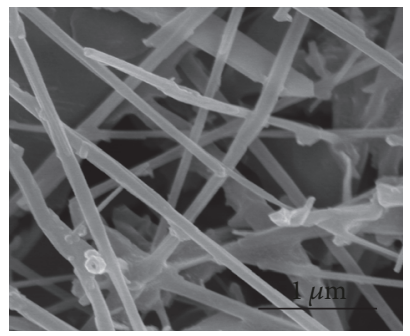

(a)

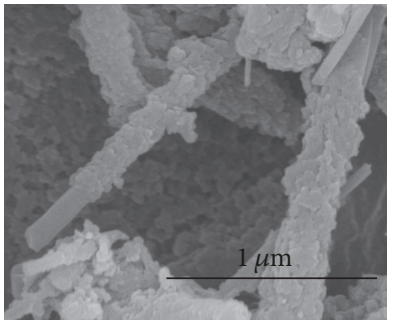

(b)

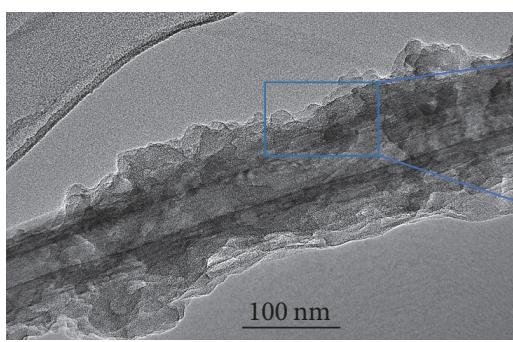

(c)

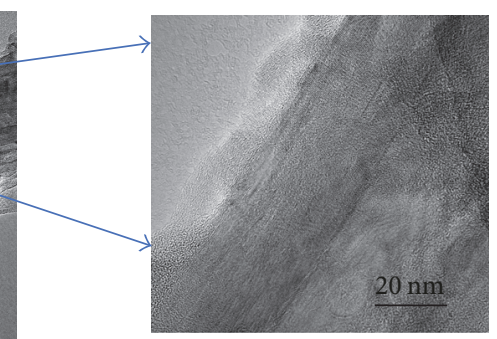

(d)

FIgure 10: (a) SEM imaging of BNNTs. (b) SEM imaging of $\mathrm{NH}_{2}$ Ph-BNNTs. (c) TEM imaging of $\mathrm{NH}_{2}$ Ph-BNNTs. (d) High resolution TEM images of $\mathrm{NH}_{2} \mathrm{Ph}-\mathrm{BNNTs}$.

modified BNNTs could be used as filler to directly mix with polymer matrix and prepare nanocomposites. The reactive aniline groups decorated upon the surface of BNNTs would contribute to the potential applications and further chemical design.

\section{Conflicts of Interest}

The authors declare that there are no conflicts of interest regarding the publication of this article.

\section{Acknowledgments}

The authors acknowledge financial support from the National Natural Science Foundation of China (Grant no. 51403216) and PetroChina Innovation Foundation. This paper was sponsored by the Seed Foundation of Innovation and Creation for Graduate Students in Northwestern Polytechnical University (Grant no. 2017207).

\section{References}

[1] S. Iijima, "Helical microtubules of graphitic carbon," Nature, vol. 354, no. 6348, pp. 56-58, 1991.

[2] C. W. Chang, W.-Q. Han, and A. Zettl, "Thermal conductivity of B-C-N and BN nanotubes," Applied Physics Letters, vol. 86, no. 17, Article ID 173102, pp. 1-3, 2005.

[3] J. S. Lauret, R. Arenal, F. Ducastelle et al., "Optical transitions in single-wall boron nitride nanotubes," Physical Review Letters, vol. 94, no. 3, Article ID 037405, 2005.

[4] S. Berber, Y.-K. Kwon, and D. Tománek, "Unusually high thermal conductivity of carbon nanotubes," Physical Review Letters, vol. 84, no. 20, pp. 4613-4616, 2000.
[5] Z. Gao, C. Zhi, Y. Bando, D. Golberg, and T. Serizawa, "Noncovalent functionalization of disentangled boron nitride nanotubes with flavin mononucleotides for strong and stable visiblelight emission in aqueous solution," ACS Applied Materials \& Interfaces, vol. 3, no. 3, pp. 627-632, 2011.

[6] L. Fu, T. Wang, J. Yu et al., "An ultrathin high-performance heat spreader fabricated with hydroxylated boron nitride nanosheets," 2D Materials, vol. 4, no. 2, Article ID 025047, 2017.

[7] C. W. Chang, A. M. Fennimore, A. Afanasiev et al., "Isotope effect on the thermal conductivity of boron nitride nanotubes," Physical Review Letters, vol. 97, no. 8, Article ID 085901, 2006.

[8] X. D. Bai, D. Golberg, Y. Bando et al., "Deformation-driven electrical transport of individual boron nitride nanotubes," Nano Letters, vol. 7, no. 3, pp. 632-637, 2007.

[9] F. Cao, W. Ren, Y.-M. Ji, and C. Zhao, “The structural and electronic properties of amine-functionalized boron nitride nanotubes via ammonia plasmas: A density functional theory study," Nanotechnology, vol. 20, no. 14, Article ID 145703, 2009.

[10] W. L. Wang, Y. Bando, C. Y. Zhi, W. Y. Fu, E. G. Wang, and D. Golberg, "Aqueous noncovalent functionalization and controlled near-surface carbon doping of multiwalled boron nitride nanotubes," Journal of the American Chemical Society, vol. 130, no. 26, pp. 8144-8145, 2008.

[11] J. Wang, C. H. Lee, and Y. K. Yap, "Recent advancements in boron nitride nanotubes," Nanoscale, vol. 2, no. 10, pp. 2028-2034, 2010.

[12] G. Ciofani, S. Danti, D. D’Alessandro, S. Moscato, and A. Menciassi, "Assessing cytotoxicity of boron nitride nanotubes: interference with the MTT assay," Biochemical and Biophysical Research Communications, vol. 394, no. 2, pp. 405-411, 2010.

[13] A. Maguer, E. Leroy, L. Bresson, E. Doris, A. Loiseau, and C. Mioskowski, "A versatile strategy for the functionalization of boron nitride nanotubes," Journal of Materials Chemistry, vol. 19, no. 9, pp. 1271-1275, 2009. 
[14] Q. Huang, A. S. D. Sandanayaka, Y. Bando et al., "Donor-acceptor nanoensembles based on boron nitride nanotubes," Advanced Materials, vol. 19, no. 7, pp. 934-938, 2007.

[15] M. D. Esrafili, "A comparative study on carbon/silicon doping effects on electronic structure and surface electrostatic potential of $(6,0)$ boron-nitride nanotube: A DFT investigation," Structural Chemistry, vol. 24, no. 4, pp. 1207-1214, 2013.

[16] W. An, X. Wu, J. L. Yang, and X. C. Zeng, "Adsorption and surface reactivity on single-walled boron nitride nanotubes containing stone-wales defects," The Journal of Physical Chemistry C, vol. 111, no. 38, pp. 14105-14112, 2007.

[17] F. L. Cao, W. Ren, Y.-M. Ji, and C. Y. Zhao, "The structural and electronic properties of amine-functionalized boron nitride nanotubes via ammonia plasmas: A density functional theory study," Nanotechnology, vol. 20, no. 14, Article ID 145703, 2009.

[18] T. Sainsbury, T. Ikuno, D. Okawa, D. Pacilé, J. M. J. Fréchet, and A. Zettl, "Self-assembly of gold nanoparticles at the surface of amine- and thiol-functionalized boron nitride nanotubes," The Journal of Physical Chemistry C, vol. 111, no. 35, pp. 12992-12999, 2007.

[19] G. Ciofani, G. G. Genchi, I. Liakos et al., "A simple approach to covalent functionalization of boron nitride nanotubes," Journal of Colloid and Interface Science, vol. 374, no. 1, pp. 308-314, 2012.

[20] T. Ikuno, T. Sainsbury, D. Okawa, J. M. J. Fréchet, and A. Zettl, "Amine-functionalized boron nitride nanotubes," Solid State Communications, vol. 142, no. 11, pp. 643-646, 2007.

[21] T. Sainsbury, A. Satti, P. May et al., "Oxygen radical functionalization of boron nitride nanosheets," Journal of the American Chemical Society, vol. 134, no. 45, pp. 18758-18771, 2012.

[22] C. Y. Zhi, Y. Bando, W. L. Wang, C. C. Tang, H. Kuwahara, and D. Golberg, "Molecule ordering triggered by boron nitride nanotubes and "green" chemical functionalization of boron nitride nanotubes," The Journal of Physical Chemistry C, vol. 111, no. 50, pp. 18545-18549, 2007.

[23] X. J. Dai, Y. Chen, Z. Chen et al., "Controlled surface modification of boron nitride nanotubes," Nanotechnology, vol. 22, no. 24, Article ID 245301, 2011.

[24] L. Q. Xu, Y. Y. Peng, Z. Y. Meng et al., "A co-pyrolysis method to boron nitride nanotubes at relative low temperature," Chemistry of Materials, vol. 15, no. 13, pp. 2675-2680, 2003.

[25] Q. Huang, Y. Bando, C. Zhi et al., "Chemical peeling and branching of boron nitride nanotubes in dimethyl sulfoxide," Angewandte Chemie International Edition, vol. 45, no. 13, pp. 20442047, 2006.

[26] R. Sundaram, S. Scheiner, A. K. Roy, and T. Kar, "Site and chirality selective chemical modifications of boron nitride nanotubes (BNNTs) via Lewis acid-base interactions," Physical Chemistry Chemical Physics, vol. 17, no. 5, pp. 3850-3866, 2015.

[27] W. Lei, V. N. Mochalin, D. Liu, S. Qin, Y. Gogotsi, and Y. Chen, "Boron nitride colloidal solutions, ultralight aerogels and freestanding membranes through one-step exfoliation and functionalization," Nature Communications, vol. 6, article no. 8849, 2015.

[28] W. Jin, W. Zhang, Y. Gao, G. Liang, A. Gu, and L. Yuan, "Surface functionalization of hexagonal boron nitride and its effect on the structure and performance of composites," Applied Surface Science, vol. 270, pp. 561-571, 2013.

[29] D. Kim, H. Muramatsu, and Y. A. Kim, "Hydrolytic Unzipping of Boron Nitride Nanotubes in Nitric Acid," Nanoscale Research Letters, vol. 12, no. 1, article no. 94, 2017. 


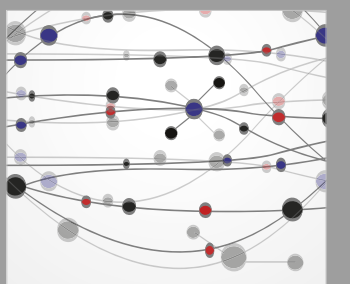

The Scientific World Journal
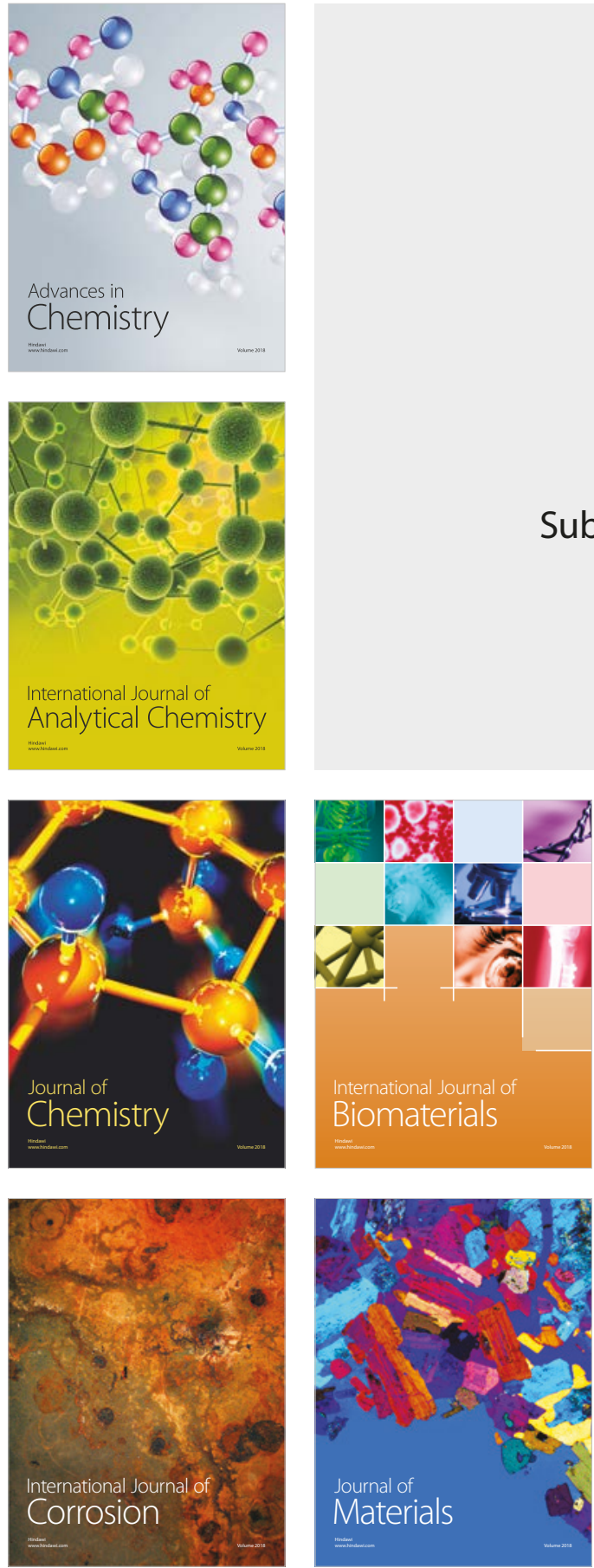

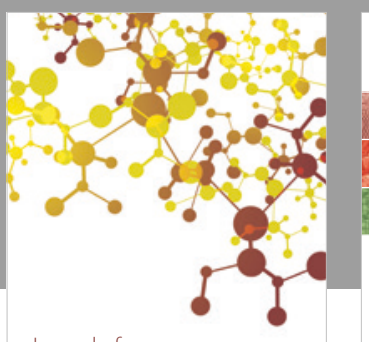

Journal of

Applied Chemistry
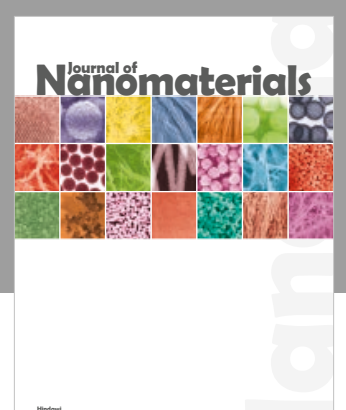

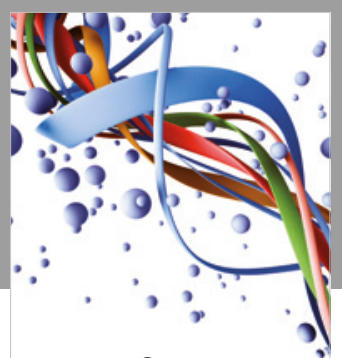

Scientifica

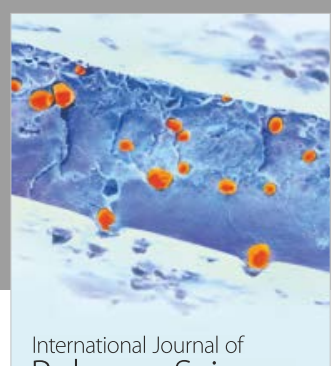

Polymer Science

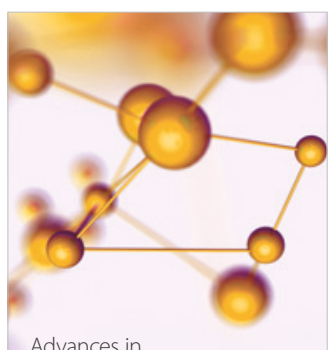

Physical Chemistry
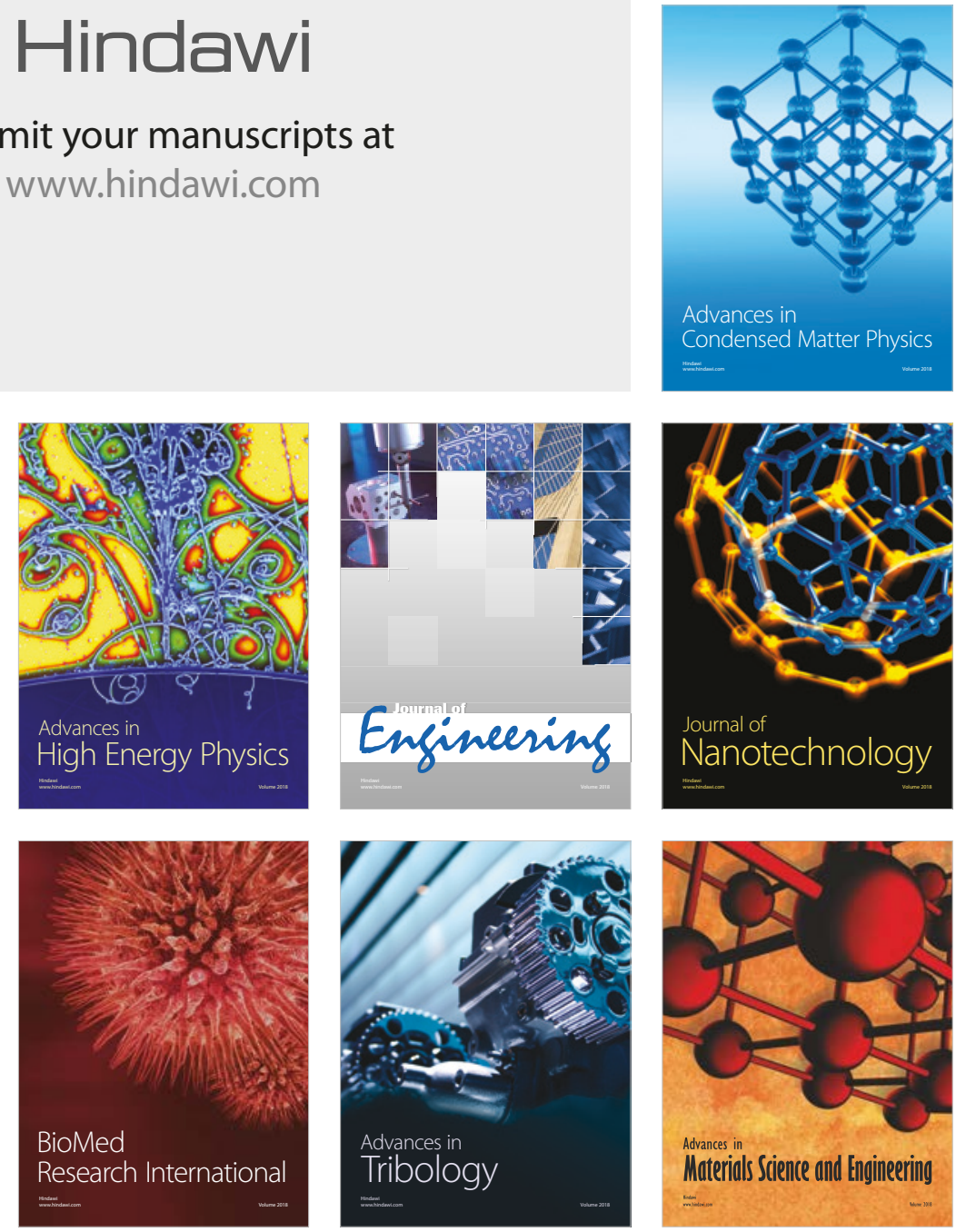